\title{
Successful Management of Parsonage-Turner Syndrome with Steroids in the Post-acute Weakness Phase: A Case Report
}

\author{
Authors: \\ Mehmood Ahmed Butt, ${ }^{1}$ Kuchalambal Agadi, ${ }^{2}$ Manju Mehmood Butt, \\ *Maliha Mehmood Butt ${ }^{2}$ \\ 1. Ahmadiyya Mission Hospital, Kaleo, Ghana \\ 2. Department of Clinical and Translational Research, Larkin Community Hospital, \\ South Miami, Florida, USA \\ *Correspondence to malihabutt1993@gmail.com \\ Disclosure: The authors have declared no conflicts of interest. \\ Received: \\ 15.03 .21 \\ Accepted: \\ 08.07.21 \\ Keywords: \\ Adjuvant therapy, autoimmune disease, brachial nerves, brachial plexus, laryngeal \\ nerve, Parsonage-Turner Syndrome (PTS), phrenic nerve, steroids. \\ Citation: \\ EMJ Neurol. 2021;9[1]:81-87.
}

\section{Abstract}

Introduction: Parsonage-Turner Syndrome (PTS) is a rare disease of the brachial plexus of unclear aetiology. The limited data available typically describes involvement of branches of brachial nerves. The authors present a case of PTS with a rare combination of unilateral brachial plexus, phrenic nerve, and recurrent laryngeal nerve injuries. They also highlight successful treatment with pharmacological intervention despite several months' delay in diagnosis. The 35-year-old female presented with acute onset of severe left shoulder pain followed by severe progressive weakness of the left shoulder muscles, progressive weakness of her voice, nasal regurgitation of fluids, paroxysmal bouts of coughing, and exertional dyspnoea at rest. The symptoms remained undiagnosed for about 10 months. A clinical diagnosis of exclusion of PTS was finally made, and treatment with steroids, neurotropic drugs, and physiotherapy was started. The patient has recovered significantly since then and continues to improve.

Conclusion: The authors presented a case of PTS with a rare combination of brachial plexus, recurrent laryngeal nerve, and phrenic nerve injuries. This case was also remarkable for the significant improvement in her symptoms with treatment, despite the delay in diagnosis. This bears evidence that steroids and adjuvant therapy is useful even months after onset of the disease.

\section{INTRODUCTION}

Parsonage-Turner Syndrome (PTS) is a rare autoimmune disease of the peripheral nervous system. It is thought to occur in 1-3 people in 100,000; however, this incidence might be an underestimation because of underdiagnosis. ${ }^{1,2}$ This condition, also known as brachial plexopathy, is centred around inflammatory damage to

specific peripheral nerve bundles as evidenced by histopathologic and neurophysiologic studies (see Diagnostic Tests). While the typical presentation of PTS involves brachial plexus, other nerves like the lumbosacral plexus, phrenic nerves, and recurrent laryngeal nerves are rarely affected. The authors' case is only the second report in the medical literature that shows this unique combination of brachial plexus, phrenic nerve, and recurrent laryngeal nerve palsies. ${ }^{3}$ 


\section{CASE DESCRIPTION}

A 35-year-old female presented with pain and severe progressive weakness of the left shoulder. Her symptoms started about 10 months earlier as severe acute pain in the left shoulder, jaw, and chest. She had undergone a dental surgery for dental caries 2 weeks before the symptoms started. On Day 7 of symptom onset, she noticed gradual resolution in pain as well as onset of progressive weakness of the shoulder evidenced by drooping of the shoulder and weakness in lifting the arm above shoulder level, but no noticeable weakness of the forearm or hand. The symptoms were accompanied by a change in her voice, described as high-pitched and low volume, as well as a paroxysmal cough and breathlessness. She also reported regurgitation of liquids through her nose right after swallowing and difficulty swallowing liquids "unless she pushed through the left side of her throat."

Her symptoms remained undiagnosed for months and were treated unsuccessfully as laryngitis, tonsillitis, and pneumonia with nonsteroidal anti-inflammatory drugs (NSAIDs) and antibiotics, which included 5-day courses of oral amoxicillin-clavulanic acid and oral azithromycin sequentially.
On presentation at the authors' hospital 10 months after the onset of symptoms, she appeared weak, anxious, and spoke in a low volume. She was breathless at rest and found it difficult to speak in complete sentences. Her left shoulder was drooping, with deltoid and supraspinatus muscles noticeably atrophied. There was no medial scapular winging that suggested that the long thoracic nerve was spared. The passive range of motion at the neck and shoulder joints was normal and not associated with the pain. Muscle strength on the left shoulder extension was $4 / 5$, suggesting possible involvement of latissimus dorsi, teres major and posterior deltoid, and left shoulder abduction up to $90^{\circ}$ was $3 / 5$, indicating involvement of supraspinatus and deltoid muscles. Muscle strength on flexion and extension of the elbow and internal and external rotation of the left arm was 5/5. Muscle strength assessment on the right upper limb was normal. Touch, pain, and temperature sensation were normal in all the dermatomes, including the left $\mathrm{C} 4-\mathrm{C} 5$. The left biceps reflex was +1 , and the brachioradialis and triceps reflexes were +2 . Chest findings were significant for reduced air entry, vesicular breath sounds, and mild crepitations over the left lower lung zone. The rest of the physical examination was normal.

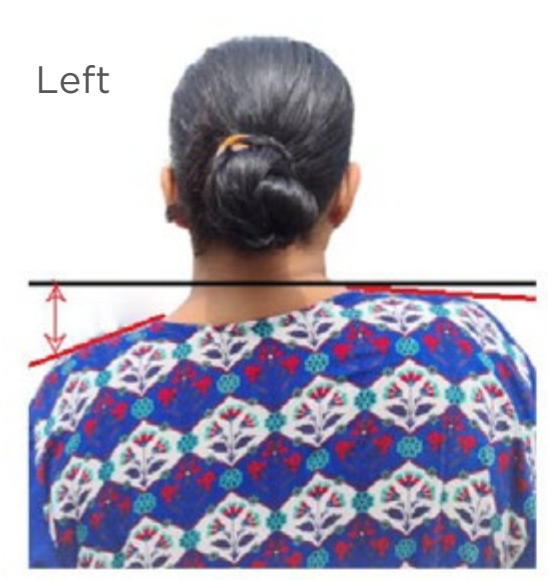

Panel a

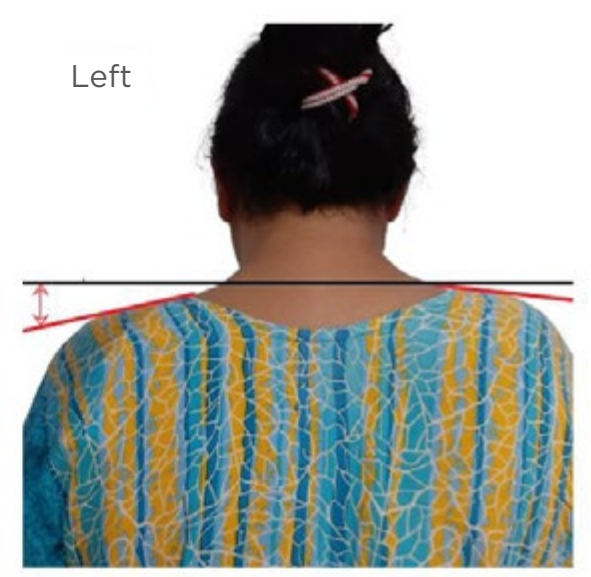

Panel b

\section{Figure 1: Appearance of patient at presentation and after treatment.}

A) Shows the patient at presentation with left shoulder drooping. The red lines follow the slope of the shoulders and show that the left shoulder slope is steeper than the right. The black horizontal line is drawn through the base of the neck to show the drop in the height of the left shoulder. B) The patient after 1 month of treatment. There is improvement in the drop of the left shoulder, as seen by the reduction in the distance from the base of the neck to the slope of the shoulder. 
Figure 1 compares the patient's appearance at presentation and after 1 month of treatment. Laboratory investigation showed normal complete blood count, comprehensive metabolic Panel, erythrocyte sedimentation rate. A cervical spine MRI showed mild intervertebral disc degeneration but with preserved disc height and normal vertebral body. There was no evidence of vertebral fracture or spondylolisthesis. The antero-posterior view of the chest radiograph was remarkable for a significant elevation of the left hemidiaphragm supporting the suspicion of left hemidiaphragm paralysis. Electromyography and nerve conduction studies could not be done in the authors' poor resource setting.

Table 1: Common differential diagnoses of Parsonage-Turner Syndrome. ${ }^{4-6}$

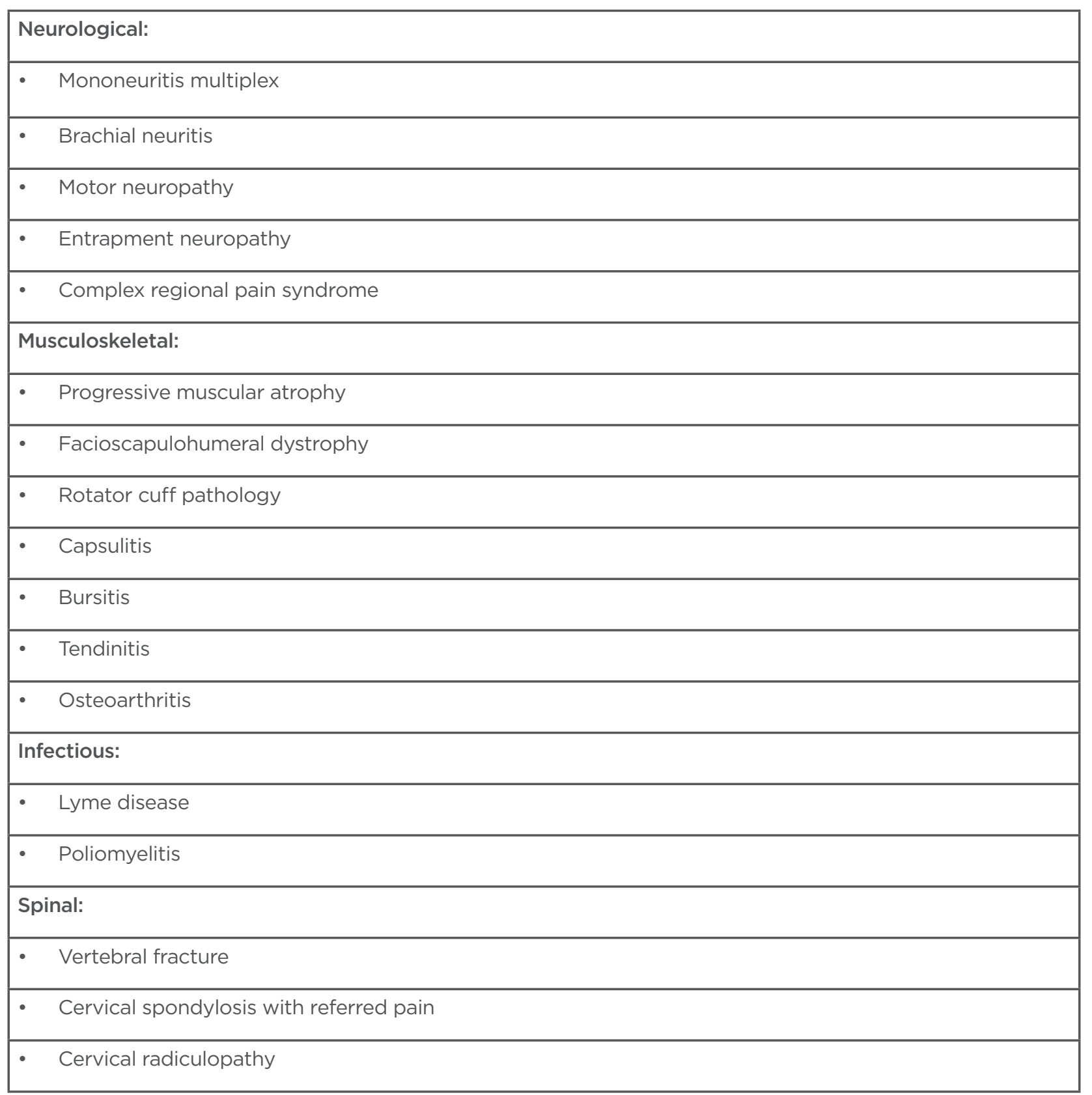


After exclusion of differential diagnoses (Table 1), a clinical diagnosis of PTS was made, and the patient was started on prednisolone tablets at $1 \mathrm{mg} / \mathrm{kg}$ per day for 4 weeks to be tapered gradually. Nootropics were given as adjuvant treatment and included Nucleo CMP Forte ${ }^{\circledast}$ (Ferrer, Barcelona, Spain) and Neuropat ${ }^{\circledR}$ (Indus Life Sciences Pvt. Ltd., Chennai, India), containing high doses of nucleotides, cyanocobalamin, alpha lipoic acid, folic acid, thiamine, and pyridoxine. The patient was started on physical therapy with incentive spirometry and shoulder strengthening exercises. Within 2 weeks, the patient experienced remarkable improvement of her condition, evidenced by better shoulder muscle strength, reduced breathlessness and cough, and improved voice quality. She continues to improve with the above management.

\section{DISCUSSION}

\section{Background}

PTS is a type of neuralgic amyotrophy, named after the two doctors, M.J. Parsonage and J.W. Aldren Turner, who reported 136 cases of this condition in 1946.4 Although it is commonly reported to occur in the brachial plexus, suprascapular nerve, long thoracic nerve, and axillary nerve, several other nerves such as anterior interosseous nerve, lateral antebrachial cutaneous nerve, superficial radial nerve, and even cranial nerves may also be involved. Both motor and sensory nerves are affected. ${ }^{5}$ However, sensory symptoms may not be present in over one-third of patients. After analysing 246 cases of PTS, van Alfen et al. mention that $62.5 \%$ develop pain during the course; presentation is unilateral and asymmetric in $71.5 \%$ and $97.0 \%$, respectively. In their analysis, they found that pain lasted longer in males than in females, but the outcome was poorer in females compared to males. They also found that in almost all cases, there were associated motor symptoms. ${ }^{6}$ Recurrent laryngeal nerve was found to be involved in up to $18.6 \%$ of patients, more common among those with hereditary neuralgic amyotrophy than idiopathic neuralgic amyotrophy. Fransz et al. note that winging of the scapula is present in two-thirds of the patients diagnosed with PTS. ${ }^{7}$ As these symptoms are non-specific, present in a variety of different neurological conditions, it is difficult to diagnose this condition. .,8 $^{6}$

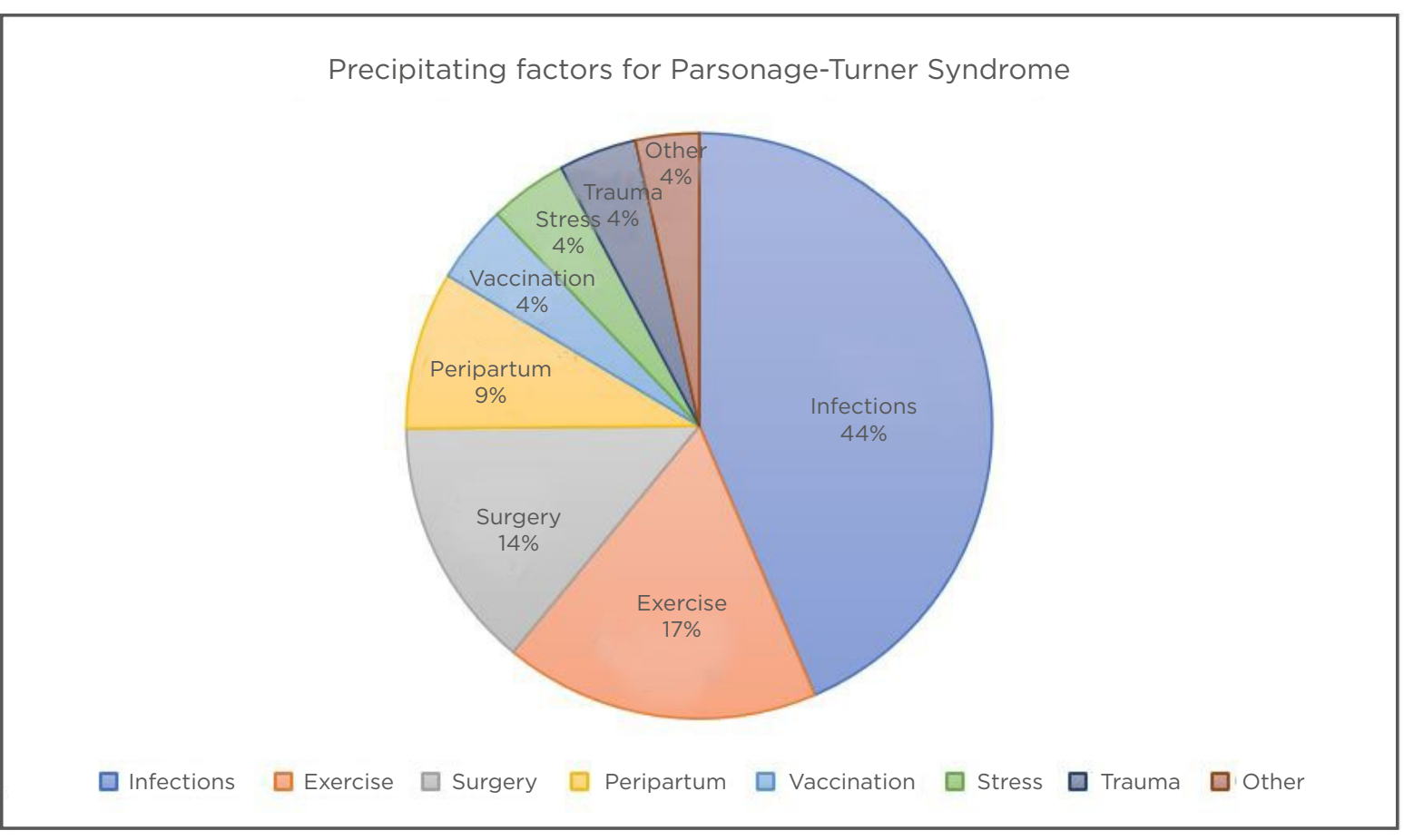

Figure 2: Pie Chart describing common precipitating factors for Parsonage-Turner Syndrome. ${ }^{4,7,10}$ 


\section{Pathophysiology}

Neurologists propose that this condition is precipitated when genetically predisposed individuals are exposed to certain precipitating events. $^{6}$ These triggers can be categorised into either infectious, accounting for about 25$55 \%$ cases, $^{9}$ environmental, or immunological, and stress either due to exercise or surgery (Figure 2). ${ }^{8}$ The authors' patient experienced the symptoms 2 weeks after a dental surgery intrapartum. While PTS precipitated by surgical procedures has been widely reported, the authors found only one other case report in the medical literature of a 28-year-old female who was diagnosed with PTS following an orthognathic surgery. The association between oral surgery and PTS is postulated to be either because of mechanical injury from traction or inadequate head and neck position, or an immune-mediated brachial nerve injury in a genetically predisposed person.." Following a precipitating event, there is a multi-focal inflammation of the affected nerves. The immune pathogenesis is evidenced by the perineural thickening, neovascularisation, and focal fibre loss seen in nerve biopsies. ${ }^{12}$ Recent studies with high resolution ultrasound of peripheral nerves in PTS supports the inflammatory process underlying the disease. The segmental swelling of axons indicates neuronal damage, while the hourglass constrictions and torsions suggest friable nerves which are further damaged by movement and abnormal positions..$^{13}$

\section{Diagnostic Tests}

PTS is a clinical diagnosis. However, investigations are important both for exclusion of differential diagnoses and as supporting evidence for the disease. Standard tests, such as blood tests, and imaging such as CT and MRI are used to exclude other diseases, some of which are listed in Table 1. In the authors' patient, basic investigations that included complete blood count, to rule out any acute infection; comprehensive metabolic panel, to explore any underlying comorbidities such as diabetes mellitus, renal, or liver dysfunction; and erythrocyte sedimentation rate as an inflammatory marker, were all normal. The chest $\mathrm{X}$-ray was done to investigate possible thoracic or neck masses compressing the brachial plexus. However, the only relevant finding on the chest $X$-ray was the marked elevation of the left hemidiaphragm, indicating a left hemidiaphragm paralysis. The cervical spine MRI was only significant for mild to moderate vertebral degeneration, but no evidence of soft tissue masses or injury was found. In the poor resource setting, electromyography and nerve conduction studies could not be done.

Diagnosis was made through a comprehensive clinical assessment. magnetic resonance neurography is a more sensitive imaging test than MRI for peripheral nervous system pathology. Its findings to be noted for PTS include thickening and diffuse high signal intensity in the brachial plexus trunks in coronal T1 weighted sequences. This is not very specific, however, because denervation can be seen in a variety of clinical settings like trauma, cervical spondylosis, neuropathy, amyotrophic lateral sclerosis, pernicious anaemia, and infections. ${ }^{14.15}$

Neurophysiologic studies are most helpful in aiding the diagnosis. They typically reveal evidence of severe plexopathy. Electromyography might show positive sharp waves and fibrillation in the acute phase, and early reinnervation with polyphasic motor unit potentials in the late phase. Nerve conduction studies reveal absent sensory nerve action potential, reduced compound motor action potentials, and prolonged $F$ response in severe disease. However, both of these studies are sensitive only 2-3 weeks after the onset of the disease. ${ }^{16}$ Due to this challenge and the varied clinical presentation of the disease, the diagnosis of PTS is often under-recognised or can be delayed. ${ }^{17}$ An emerging diagnostic modality is high resolution ultrasound, which typically shows structural nerve abnormalities like segmental swelling, hourglass constrictions, and torsions, which are very specific for PTS. ${ }^{13}$ However, its utilisation is currently limited since sufficient technological expertise is needed to accurately identify lesions.

\section{Treatment}

Most neurologists agree that though a person with PTS takes a long time to recover, conservative treatment will suffice. Ibrahim et al. suggest that physical therapy will suffice to treat the symptoms. While conservative treatment with analgesics and physical therapy is the generally accepted practice, studies done in recent years substantiate the efficacy of steroid use in the acute phase of the disease. In a case 
series by van Alfen et al, addition of prednisolone $1 \mathrm{mg} / \mathrm{kg}$ per day in the treatment regimen of a cohort of patients with PTS during the acute phase of illness promoted recovery of patients compared to the untreated cohort, shortening the duration of pain and time until the onset of recovery from paresis (28\% within the first month versus $6.3 \%$ in the untreated group; $p=0.001) .{ }^{18}$ In a few other studies too, oral and intravenous steroids and immunotherapy in the acute painful phase are seen to improve symptoms and accelerate recovery. 2,19,20 There has been a paucity of data on the use of steroids in the acute phase but especially in the post-acute weakness phase of PTS. Most of the studies done on PTS involve patients who presented and are treated within 1 month of disease onset. Lee et al. evaluated the effect of injected and oral prednisolone given at an approximate dose of $1 \mathrm{mg} / \mathrm{kg}$ per day to a group of six patients with PTS who presented in the post-acute weakness phase of the disease. They reported faster muscle strength recovery and return to work in the steroid group compared with a cohort treated with NSAIDs and physical therapy but no steroids. ${ }^{21}$

The authors used a similar treatment regime seen in most of the studies involving PTS management on their patient, with tapered $1 \mathrm{mg} / \mathrm{kg}$ per day dose of prednisone over 2-4 weeks',18,19 They also used nucleotides and vitamins as adjuvant therapy. While there has been no evidence of their use in PTS, use of these adjuvants, sometimes called nootropics, has been found beneficial in the treatment of peripheral neuropathies, neuromuscular diseases, and brain injuries..$^{22,23}$

While the authors noted marked improvement in their patient after starting the treatment regimen (NSAIDs, prednisone, nootropics, and physical therapy), they cannot conclude which of the treatments was the most relevant in the resolution of the symptoms. It should also be considered the recovery period of PTS generally ranges from 1-3 years, making it difficult to exclude the natural course and resolution of the disease in their patient, who presented to them 10 months after disease onset. Large scale randomised clinical trials with different treatment modalities will be helpful in determining efficacy of therapies. However, the relatively low prevalence of the disease makes it difficult to conduct such studies. Surgical treatment with decompression and micro-neurolysis is also useful in patients with persistent symptoms. ${ }^{17}$

\section{Outcome}

An electrodiagnostic study of natural history of the disease on 26 patients with PTS showed complete recovery in about one-half of the patients at 1 year. ${ }^{24}$ However, clinical studies vary in this assessment. Earlier studies suggest that about two-thirds of the patients have residual persisting pain and functional impairment for up to 3 years of follow-ups with subjective full recovery from symptoms only seen in $<10 \%$ at 3 years. ${ }^{25}$ More recent studies that evaluate the use of analgesics, steroids, and physical therapy have shown better recovery rates and return to functionality. $6,20,26$

\section{SUMMARY}

The authors present here a case of PTS with a rare involvement of brachial plexus, recurrent laryngeal nerve, and phrenic nerve. This case was remarkable for the significant improvement in her symptoms with their treatment regimen, despite the lateness of the presentation. This bears evidence that steroids and adjuvant therapy is useful even months after onset of the disease. Further larger, randomised trials will be required to establish the effectiveness of steroids in the weakness phase of PTS.

\section{References}

1. Ashworth NL. Brachial Neuritis. 2020. Available at: http://emedicine. medscape.com/article/315811overview. Last accessed: 28 February 2021.

2. Feinberg JH, Radecki J. Parsonageturner syndrome. HSS J.

\section{0;6(2):199-205.}

3. Chen YM et al. Bilateral neuralgic amyotrophy presenting with left vocal cord and phrenic nerve paralysis. $J$ Formos Med Assoc. 2007;106(8):6804.

4. Parsonage MJ, Turner JW. Neuralgic amyotrophy; the shoulder girdle syndrome. Lancet. 1948;1(6513), 973-8.

5. van Alfen N. Clinical and pathophysiological concepts of neuralgic amyotrophy. Nat Rev Neurol. 2011;7(6):315-22. 
6. van Alfen N, van Engelen BG. The clinical spectrum of neuralgic amyotrophy in 246 cases. Brain. 2006;129(Pt 2):438-50.

7. Fransz DP et al. Parsonage-Turner syndrome following post-exposure prophylaxis. BMC Musculoskelet Disord. 2014;15:265.

8. Tsairis P et al. Natural history of brachial plexus neuropathy. Report on 99 patients. Arch Neurol. 1972;27(2):109-17.

9. Ibrahim R et al. Atypical presentation of Parsonage-Turner Syndrome. Cureus 2020;12(6):e8892.

10. Stek CJ et al. Neuralgic amyotrophy associated with Bartonella henselae infection. J Neurol Neurosurg Psychiatry. 2011;82(6):707-8.

11. Huang $Y C$ et al. Postsurgical brachial neuritis after orthognathic surgery: a case report. J Oral Maxillofac Surg. 2005;63(9):1387-90.

12. Staff NP et al. Immune-mediated postsurgical neuropathy. Abstract M-62. American Neurological Association 134th Annual Meeting Poster Session Abstracts, 12 October 2009.

13. Gstoettner $\mathrm{C}$ et al. Neuralgic amyotrophy: a paradigm shift in diagnosis and treatment. $\mathrm{J}$
Neurol Neurosurg Psychiatry. 2020;91(8):879-88.

14. Bongers KS et al. Skeletal muscle denervation causes skeletal muscle atrophy through a pathway that involves both Gadd45a and HDAC4. Am J Physiol Endocrinol Metab. 2013;305(7):E907-15.

15. Gaskin CM, Helms CA. ParsonageTurner syndrome: MR imaging findings and clinical information of 27 patients. Radiology. 2006;240(2):5017.

16. Yu DK et al. Neuroradiologic and neurophysiologic findings of neuralgic amyotrophy. J Korean Neurosurg Soc. 2010;48(5):423-8.

17. McCarty EC et al. Brachial neuritis Clin Orthop Relat Res. 1999:(368):3743.

18. van Alfen $\mathrm{N}$ et al. Treatment for idiopathic and hereditary neuralgic amyotrophy (brachial neuritis) Cochrane Database Syst Rev. 2009;2009(3):CD006976.

19. Wiser Smith DP et al. Intravenous corticosteroid therapy for bilateral parsonage-turner syndrome: a case report and review of the literature. Reg Anesth Pain Med. 2014:39(3):243-7.

20. Eijk JJ et al. Evaluation of prednisone treatment in the acute phase of neuralgic amyotrophy: an observational study. J

Neurol Neurosurg Psychiatry. 2009;80(10):1120-4.

21. Lee GW, Kwon YH. The purport of steroid treatment in the weakness phase of Parsonage-Turner Syndrome. J Korean Orthop Assoc. 2012;7(4):311-5.

22. Martynyuk L et al. The clinical efficacy of the nucleo CMP forte usage in the combined treatment of diabetic peripheral neuropathy. Endocrine Abstracts. 2017;49:EP420.

23. Povedano $\mathrm{M}$ et al. Observational pilot study of patients with carpal tunnel syndrome treated with Nucleo CMP Forte $^{T M}$. Pain Manag. 2019:9(2):123-9.

24. Feinberg $\mathrm{JH}$ et al. The electrodiagnostic natural history of parsonage-turner syndrome. Muscle Nerve. 2017;56(4):737-43

25. van Alfen $\mathrm{N}$ et al. Long-term pain, fatigue, and impairment in neuralgic amyotrophy. Arch Phys Med Rehabil. 2009;90(3):435-9.

26. Conway RR. Neuralgic amyotrophy: uncommon but not rare. Mo Med. 2008;105(2):168-9. 